\title{
Optimization of Human ECG Signal Acquisition Based on Wavelet Neural Network and Genetic Algorithm
}

\author{
Jian CHEN \\ College of Instrumentation \& Electrical Engineering \\ Jilin University \\ Changchun, China \\ E-mail: 18143143214@163.com \\ Yingqi LIU \\ College of Instrumentation \& Electrical Engineering \\ Jilin University \\ Changchun, China \\ E-mail: 769003678@qq.com
}

\author{
Dongmei LIANG* \\ College of Instrumentation \& Electrical Engineering \\ Jilin University \\ Changchun, China \\ E-mail: 1361946665@qq.com \\ +* Corresponding author
}

\author{
Jie LIU \\ College of Instrumentation \& Electrical Engineering \\ Jilin University \\ Changchun, China \\ E-mail: 644675796@qq.com
}

\begin{abstract}
Human ECG signal is weak, the signal-to-noise ratio is small, the collected ECG signal amplification and filtering are often accompanied by interference, the interference from the body's own interference such as EMG interference, but also from outside interference such as $50 \mathrm{~Hz}$ power frequency interference[2]. These disturbances degrade the signal-to-noise ratio of the system, and can even overwhelm the useful weak ECG signal, especially $50 \mathrm{~Hz}$ power frequency interference. If not to eliminate to a very small extent, it will affect the subsequent signal processing, diagnosis, recognition accuracy. In addition the ECG signal also includes the following two kinds of interference: First, baseline drift [3]. Usually caused by human respiration and excitement of the heart and legs, the body's movement will lead to a certain amount of baseline drift, the frequency is lower than $1 \mathrm{~Hz}$, the performance of the slow changes in the curve; Second, EMG interference, which is caused by human body tremor, its frequency range is very wide. Usually between $5 \sim 2 \mathrm{kHz}[1]$, the performance of the rapid changes in irregular waveforms. In addition, due to the test sex, age and skin conductivity, so there are differences and the impact of equipment, system acquisition of different people's ECG signal is also very different. Therefore, in order to eliminate these interference and obtain an effective ECG signal, this paper presents a genetic neural network based on human heart ECG parameters acquisition system. By combining the nonlinear approximation ability of wavelet function with the self-learning characteristic of neural network, the output accuracy of sensor can be effectively improved, the effect of non-target parameter on detection result can be eliminated, and the detection accuracy of human physiological parameters can be improved. Through the experimental test, the ECG parameters of the device are accurate and reliable data has a good practical value.
\end{abstract}

Keywords-ECG signal; frequency interference; baseline drift;

EMG interference; wavelet analysis

\section{INTRODUCTION}

Heart disease is harmful to human health, threatening people's lives, so people have been committed to the analysis of heart disease research. The diagnosis of heart disease is mainly based on a series of waveforms in the ECG amplitude and duration of some information as the basis for information on cardiac conditions mainly in these areas.ECG signal is often accompanied by the existence of interference noise in the process of acquisition [7], which makes the ECG signal blur distortion, which is not conducive to the analysis of signal morphology.

The ECG signal is a random signal. Is composed of $\mathrm{P}$, QRs, $\mathrm{T}$ wave and rest period, the wave has different frequency characteristics, simply from the time domain or frequency domain analysis is not comprehensive, can not reflect the overall characteristics of the ECG signal[5]. Only with the combination of simple time domain, frequency domain analysis of the comprehension and time-domain analysis of the comprehensive, comparative study in order to objectively grasp the characteristics of ECG waveforms [4].

Wavelet transform is a new theory developed in the late 1980 s, its function is similar to the classical Fourier transform, but the wavelet analysis method is a new signal analysis method, with multi-resolution analysis features, can better highlight the local characteristics of the signal, It is suitable for the analysis of most unsteady signals. It is also applicable to the signals with fractal structure, which is of great significance in weak random signal analysis with strong background noise. Based on the nonlinear approximation ability of wavelet function and the selflearning characteristic of neural network, a wavelet neural network suitable for high-dimensional input is proposed [9]. The activation function of hidden neurons in the neural network is wavelet basis function. Because the wavelet function is a fast decay of the local function. Therefore, the wavelet network and the RBF network belong to the same 
local approximation network; obviously have convergence speed, easy to adapt to the new sample, can avoid the advantages of large extrapolation error. Wavelet neural networks are an extension of feedforward neural networks, so a variety of training algorithms can also be used to train wavelet neural networks [10]. However, the conventional gradient method has the disadvantages of slow training speed and being easily trapped in local minimum points. Genetic algorithm is a global optimization method, which can effectively avoid the impact of local input and noise.

In order to make the wavelet neural network have higher learning precision and faster convergence speed, the genetic algorithm and wavelet neural network are combined. We propose a genetic wavelet neural network. The genetic wavelet neural network is used to realize the ECG signal acquisition, which can effectively improve the output accuracy of the sensor and eliminate the influence of the non-target parameter on the sensor output. It has a good popularization and practical value.

\section{Genetic Wavelet Neural Network Structure}

Wavelet network is a neural network model based on wavelet analysis, that is, using non-linear wavelet basis to replace the usual Sigmuld function. Symmetric wavelets form a radial basis function family by stretching parameters. The signal representation is achieved by linearly superimposing the selected wavelet base, and its network topology:

$$
\hat{f}(x)=\sum_{i=1}^{m} \omega_{i} \phi\left(\sum_{j=1}^{n} \omega_{i j} x_{j}\right)
$$

Publicity here omitted, change the format to write, draw.

The network parameters $\omega_{i}, \omega_{i j}$ can be optimized by finding the minimum of the energy function:

$$
E=\frac{1}{2} \sum_{i=1}^{N}\left[f\left(X_{i}\right)-\hat{f}\left(X_{i}\right)\right]^{2}
$$

Where $X_{i}$ denotes the training sample in group $\mathrm{t}$, $f\left(X_{i}\right)$ denotes the expected output corresponding to the $\mathrm{t}-\mathrm{th}$ training sample as input, $\hat{f}\left(X_{i}\right)$ Indicates the actual output corresponding to the $\mathrm{t}$-th training sample as input.

By using the genetic small crossing neural network for multi-sensor information fusion technology, we should first select the appropriate wavelet network model according to the requirements of the system and the characteristics of the sensor, and then use the genetic algorithm based on the existing multi-sensor information and system fusion knowledge, to establish the neural network system for offline learning, determine the right threshold of the network connection finally, the trained network is used for the actual information fusion. The signals detected by the sensor are processed as the input of the neural network by the preprocessing unit such as rectification, optimization, denoising and normalization. The neural network continually learns the new knowledge of the information fusion from the practical application, adjusts its own weight, completes the input non-linear mapping of the input space to the output space. The final use of $n$ sensors in the performance of the differences and complementarity to make up for a single sensor defects, comprehensive analysis of the surrounding environment to the correct analysis. Improve the reliability of information fusion.

\section{ECG Signal Processing BASEd ON GeNETIC WAVELET NEURAL NETWORK}

ECG signal is a kind of uneven and extremely weak electrical signal collected on the human body surface, which is susceptible to the noise signal generated by the human body itself, the instrument used and the external environment. Through the analysis of the source of the interference noise, noise can be divided into three kinds: baseline drift, power frequency interference and EMG.

(1) baseline drift: baseline drift is caused by the poor contact between the collector electrode and the body surface and the human body to produce a lower frequency of noise, the frequency is usually below $1 \mathrm{~Hz}[8]$, its performance and low frequency sine Wave similar to the slow change in the curve, the noise easily lead to ECG signal baseline position of the upper and lower drift, in particular, the accurate positioning of the ST segment have a serious impact.

(2) Frequency interference: frequency interference is generated by the signal acquisition process in the power supply network environment and ECG collection equipment generated by a kind of electromagnetic interference, the frequency of $50 / 60 \mathrm{~Hz}$ cited, the performance of $50 / 60 \mathrm{~Hz}$ sine wave and its harmonic curve, The noise and ECG signal frequency overlap, will make the ECG waveform distortion, in the waveform detection, especially for the weak $\mathrm{P}, \mathrm{T}$ wave accurate identification of interference.

(3) Electromyography: Myoelectric interference is mainly caused by human body fibrillation and human activities caused by a noise, the frequency is mainly distributed in the $5-2 \mathrm{KHz}$ and serious EMG interference and distribution in the $10 \sim 300 \mathrm{~Hz}$. Compared with the frequency segment where the ECG signal is located, the EMG interference is a high frequency signal, which is an irregular and rapidly changing waveform with Gaussian noise. The noise coincides with the serious frequency of the ECG signal. Submerged changes in the waveform of ECG signals.

The presence of interfering noise makes the collected ECG signal have a low signal to noise ratio, and sometimes it can cause serious distortion of some waveforms in the signal. This is not conducive to the detection and recognition of the characteristic parameters in the subsequent signals. In the application of clinical medicine has caused trouble. Therefore, ECG signal interference noise removal has become the ECG signal analysis of the primary problem.

\section{A. Frequency Interference Filtering}

According to the sine wave waveform, the sum of any $n$ points in the week is zero. Filter algorithm formula is to take $\mathrm{n}$ sampling points of the average, namely: 


$$
Y=\frac{1}{n} \sum_{i=1}^{n} X_{i}=\bar{X}
$$

$\mathrm{X}$ : original ECG signal, y: filtered ECG signal.

$\mathrm{F}$ is the frequency of the sine wave to be eliminated, this is $50 \mathrm{~Hz} ; \mathrm{n}$ must be an integer, otherwise it is adjusted by changing $\mathrm{s}$ ). When $\mathrm{n}$ is $\mathrm{s} / \mathrm{f}$ ( $\mathrm{s}$ is the number of sampling points per second; $f$ is the frequency of the sine wave to be eliminated At this point the rl point of interference is just a week in the $\mathrm{n}$ points, and its zero. So the interference wave is completely eliminated. The sampling frequency of the monitor is $250 \mathrm{~Hz}$, that is, 250 points per second, in order to eliminate the mixed $50 \mathrm{~Hz}$ power frequency interference, its 5 points $\mathrm{f}(250 / 50)$ sampling average as a filter output. In the implementation of the method will be the last 5 points and the value of the five points and save, each time a new point, and in the minus the earliest points, plus a new point, while the earliest removed; will be divided by 5 The average. Fig. 1 is the use of the filtering algorithm for human ECG signal sampling power frequency filter before and after the output control examples.
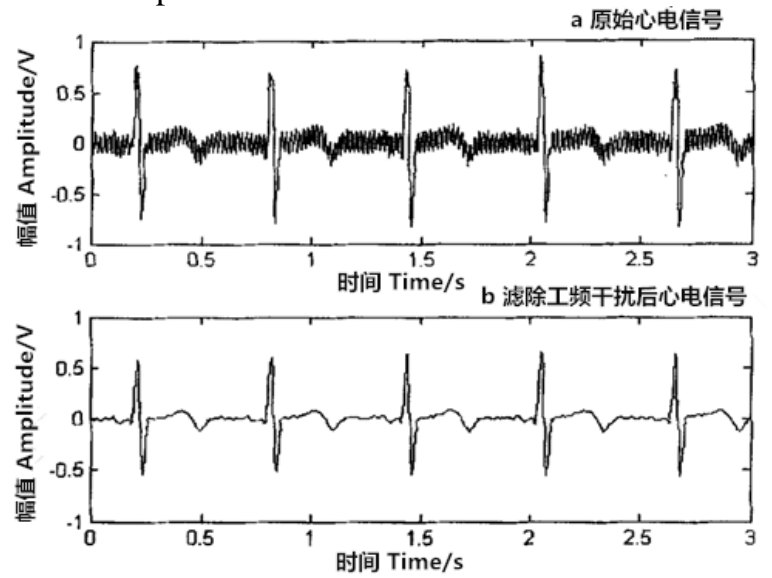

Figure 1. The ECG wave with the signal filtered and non-filtered

\section{B. Genetic Wavelet Filtering}

After removing the most significant noise from the frequency interference, the ECG signal also includes the following two kinds of interference: First, the baseline drift. Generally caused by the body breathing and excitement of the heart, the body's movement will lead to a certain amount of baseline drift, the frequency is lower than $1 \mathrm{~Hz}$, the performance of the slow change in the curve; Second, EMG interference, Meat frequency caused by it, it fishing frequency range is very wide. Generally between $5 \sim 2 \mathrm{kHz}$, the performance of irregular rapid changes in the waveform . In addition, due to the sex, age, skin conductivity and so on are different and the impact of equipment, the system of different people's ECG signal is also very different, as shown in Fig. 2, a1 to a6 is from different human In order to eliminate these disturbances, an effective ECG signal is obtained. In this paper, wavelet analysis is used to denoise the ECG signal, and the ECG signal is used to denoise the ECG signal [6].

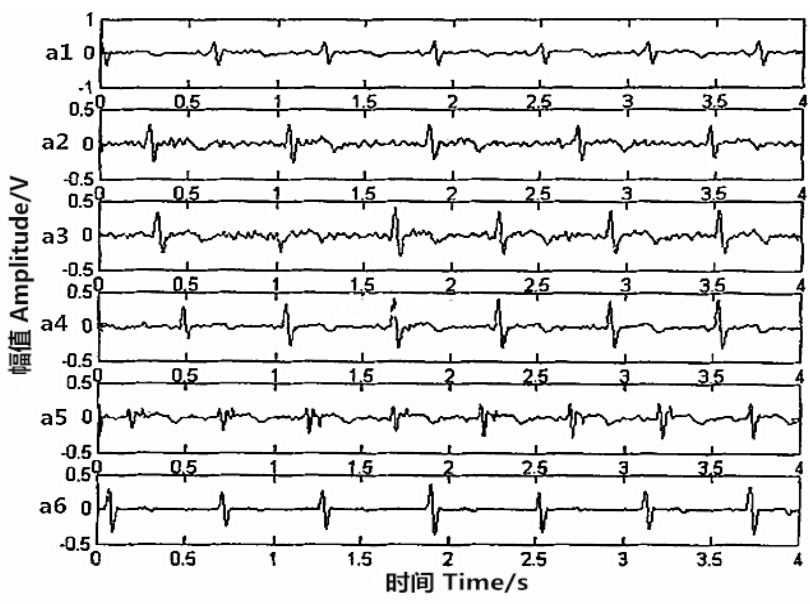

Figure 2. ECG wave of different people

A model of a noise-containing one-dimensional signal can be expressed as follows:

$$
s(i)=f(i)+\sigma \cdot e(i), i=0, \cdots, n-1
$$

$f(i)$ is the real signal, $e(i)$ is the noise, $s(i)$ is the signal containing the noise. We can use a simplest noise model to illustrate, that $s(i)$ for the Gaussian self-voice $\mathrm{N}$ $(0,1)$, the noise level is 1 . In practical applications, the useful signal is usually expressed as a low frequency signal or some relatively smooth signal, and noise is usually expressed as high frequency signal, so the denoising process can be as follows:First, the signal is decomposed by wavelet (such as three-layer decomposition, the decomposition process shown in Fig. 3, then the voice part is usually included in the approximate signal $\mathrm{CD} 1, \mathrm{CD} 2, \mathrm{CD} 3$, and therefore can be threshold and other forms of wavelet Coefficient processing, and then reconstruct the signal, you can achieve the purpose of denoising. In fact, the denoising of the $s(i)$ signal is the restoration of the original signal $f(i)$ from the noise-contaminated signal while suppressing the noise $e(i)$.

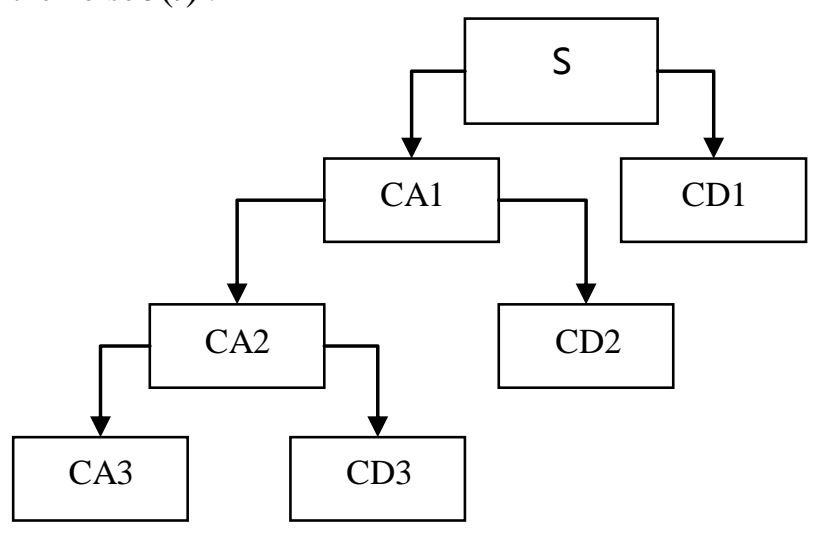

Figure 3. The process of wavelet decompose 
In general, the use of wavelet transform threshold method for denoising is divided into the following three steps [11]:

1) Orthogonal wavelet transform with noise signal: select the appropriate wavelet and wavelet decomposition level IV, the use of wavelet signal decomposition algorithm to the fourth layer, get the corresponding wavelet decomposition coefficient.

2) Threshold quantization of wavelet decomposition high frequency coefficients: a threshold value is selected for a threshold for each layer of the first to Nth layers. There are two general thresholds:

Soft threshold processing, that is, the absolute value of the signal is compared with the threshold, less than or equal to the threshold of the point becomes 0 , greater than the threshold of the point becomes the value of the difference between the value and the threshold.

Hard threshold processing, that is, the absolute value of the signal compared with the threshold, less than or equal to the threshold of the point 0 is greater than the threshold of the point remains unchanged. In general, signals processed with hard thresholds are more rough than those processed with soft thresholds.

3) Wavelet signal reconstruction: that is, the inverse wavelet transform, according to the wavelet decomposition of the low-frequency coefficients of the fourth layer and after quantization of the first to fourth layer of the high-frequency coefficient, the wavelet reconstruction, to restore the original signal estimated value.

In this experiment, the B-spline wavelet is used to denoise the ECG signal, the ECG signal is decomposed into four layers, and the corresponding wavelet decomposition coefficient is obtained. The given soft threshold method is used to denote the first to the fourth A layer of high frequency coefficients, select a threshold for quantization. And then reconstructed according to the low-frequency coefficients of the fourth layer after wavelet decomposition and the high-frequency coefficients of the first to fourth layers after quantization, and obtain the ECG signal after noise reduction. Fig. 4 shows the wavelet decomposition waveform of an ECG signal. The figure from top to bottom are the original ECG signal, wavelet decomposition of the approximate signal and the first to fourth layer of the details of the signal. It can be seen from the figure that the original ECG signal contains more obvious noise interference, and the noise component mainly concentrates on the lth and 2th detail components after wavelet decomposition. Therefore, in the process of wavelet reconstruction, Component of the scale, the use of a certain threshold operation can effectively eliminate high-frequency noise.

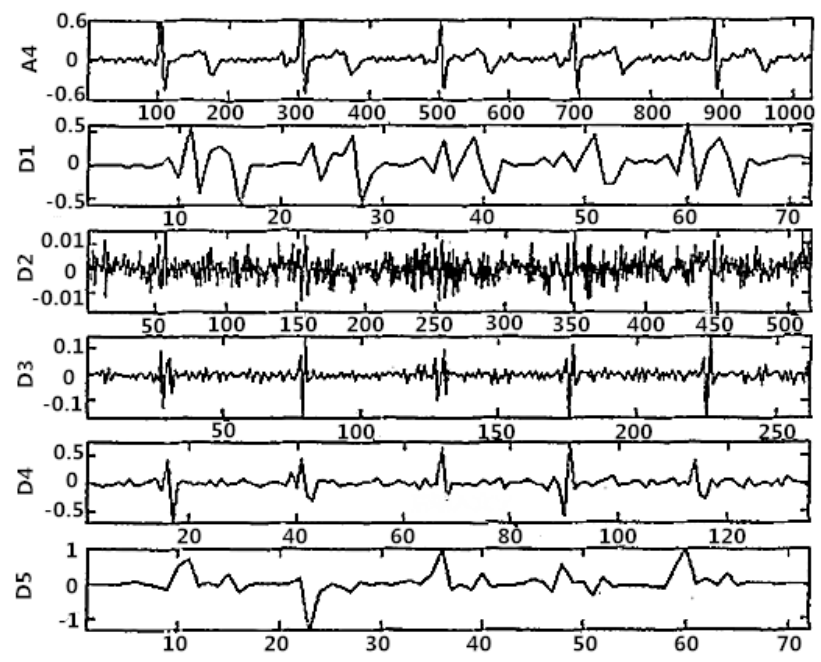

Figure 4. The ECG signal analysis of wavelet transform

Fig. 5 shows the waveforms of an ECG before and after wavelet denoising. It can be seen from the figure that the high frequency components in the original signal waveform are effectively suppressed by wavelet de-noising.
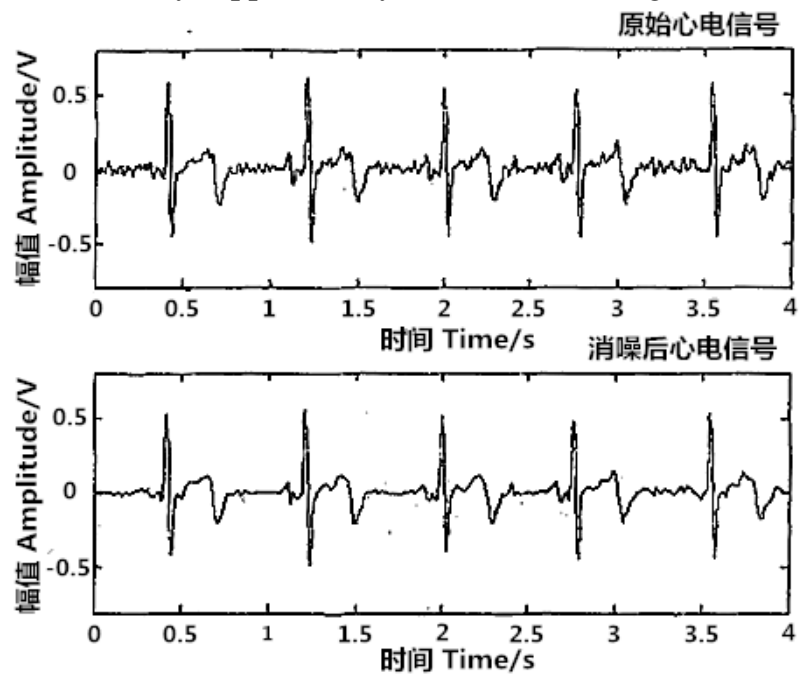

Figure 5. The ECG wave of the signal filtered ang not by wavelet transform

\section{CONCLUSION}

Genetic algorithm is used to optimize the threshold of wavelet neural network, and a neural network with genetic optimization is designed. The network is used in the genetic algorithm of genetic wavelet neural network. The system can accurately reflect the detection object and improve the quality of information collection. The results of ECG signal detection based on genetic wavelet neural network show that this method can effectively improve the output accuracy of the sensor, eliminate the influence of non - target parameters on the sensor output, and meet the requirement of ECG information acquisition. The system is designed to be simple, suitable for engineering applications, with practical value. 


\section{REFERENCES}

[1] Angela Agostinelli, Agnese Sbrollini, Corrado Giuliani, Sandro Fioretti, Francesco Di Nardo and Laura Burattini,"Segmented Beat Modulation Method for Electrocardiogram Estimation from Noisy Recordings[J], " Medical Engineering and Physics,2016.

[2] Byron H. Gottschalk, Javier Garcia-Niebla,Daniel D. Anselm,Atul Jaidka,Antoni Bayés De Luna,Adrian Baranchuk. New methodologies for measuring Brugada ECG patterns cannot differentiate the ECG pattern of Brugada syndrome from Brugada phenocopy[J]. Journal of Electrocardiology, 2016,49(2):

[3] Robin A. Ducas, Christopher Labos,David Allen, Mehrdad Golian,Maya Jeyaraman,Justin Lys,Amrinder Mann,Leslie Copstein,Sherri Vokey,Rasheda Rabbani,Ryan Zarychanski,Ahmed M. Abou-Setta,Alan H. Menkis. Pre-hospital ECG administration and clinical outcomes in ST-segment myocardial infarction: a systematic review and meta-analysis[J]. Canadian Journal of Cardiology,2016,:

[4] Jana Svehlikova, Jan Zelinka, Ljuba Bacharova,Milan Tysler. Modeling and visualization of the activation wavefront propagation to improve understanding the QRS complex changes indicating left ventricular hypertrophy[J]. Journal of Electrocardiology,2016,49(5):.

[5] Kholkhal Mourad, Bereksi Reguig Fethi. Efficient Automatic Detection Of QRS Complexes In ECG Signal Based On Reverse Biorthogonal Wavelet Decomposition And Nonlinear Filtering[J]. Measurement,2016,:
[6] Phuong Nguyen, Jong-Myon Kim. Adaptive ECG denoising using genetic algorithm-based thresholding and ensemble empirical mode decomposition[J]. Information Sciences,2016,373:.

[7] Jaspal Singh, R.K. Sharma, A.K. Gupta. A method of REM-NREM sleep distinction using ECG signal for unobtrusive personal monitoring[J]. Computers in Biology and Medicine,2016,:

[8] P.C. Bhaskar, M.D. Uplane. High Frequency Electromyogram Noise Removal from Electrocardiogram Using FIR Low Pass Filter Based on FPGA[J]. Procedia Technology,2016,25:

[9] E. Torti,D. Koliopoulos,M. Matraxia,G. Danese,F Leporati. Custom FPGA Processing for Real-Time Fetal ECG Extraction and Identification[J]. Computers in Biology and Medicine,2016,:.

[10] Xu Wang,Yongxin Zhu,Yajun Ha,Meikang Qiu,Tian Huang,Xueming $\mathrm{Si}$, Jiangxing Wu. An Energy-Efficient System on a Programmable Chip Platform for Cloud Applications[J]. Journal of Systems Architecture, 2016,:

[11] Yama Fakhri, Maria Sejersten, Mikkel Malby Schoos, Jacob Melgaard, Claus Graff, Galen S. Wagner, Peter Clemmensen, Jens Kastrup. Algorithm for the Automatic Computation of the Modified Anderson-Wilkins Acuteness Score of Ischemia from the Pre-hospital ECG in ST-segment Elevation Myocardial Infarction[J]. Journal of Electrocardiology, 2016. 